Research Article

\title{
Fuzzy Conformable Fractional Semigroups of Operators
}

\author{
Atimad Harir (D), Said Melliani, and Lalla Saadia Chadli \\ Laboratory of Applied Mathematics and Scientific Computing, Sultan Moulay Slimane University, P.O. Box 523, \\ Beni Mellal 23000, Morocco \\ Correspondence should be addressed to Atimad Harir; atimad.harir@gmail.com
}

Received 6 August 2020; Revised 16 October 2020; Accepted 21 October 2020; Published 4 November 2020

Academic Editor: Andrea Scapellato

Copyright (c) 2020 Atimad Harir et al. This is an open access article distributed under the Creative Commons Attribution License, which permits unrestricted use, distribution, and reproduction in any medium, provided the original work is properly cited.

In this paper, we introduce a fuzzy fractional semigroup of operators whose generator will be the fuzzy fractional derivative of the fuzzy semigroup at $t=0$. We establish some of their proprieties and some results about the solution of fuzzy fractional Cauchy problem.

\section{Introduction}

Fractional semigroups are related to the problem of fractional powers of operators initiated first by Bochner [1]. Balakrishnan [2] studied the problem of fractional powers of closed operators and the semigroups generated by them. The fractional Cauchy problem associated with a Feller semigroup was studied by Popescu [3]. Abdeljawad et al. [4] studied the fractional semigroup of operators. The semigroup generated by linear operators of a fuzzy-valued function was introduced by Gal and Gal [5]. Kaleva [6] introduced a nonlinear semigroup generated by a nonlinear function.In the last few decades, fractional differentiation has been used by applied scientists for solving several fractional differential equations and they proved that the fractional calculus is very useful in several fields of applications and real-life problems such as, but certainly not limited, in physics (quantum mechanics, thermodynamics, and solid-state physics), chemistry, theoretical biology and ecology, economics, engineering, signal and image processing, electric control theory, viscoelasticity, fiber optics, stochastic-based, finance, tortoise walk, Baggs and Freedman model, normal distribution kernel, time-fractional nonlinear dispersive PDEs, fractional multipantograph system, time-fractional generalized Fisher equation and time-fractional $k(m, n)$ equation, and nonlinear time-fractional Schrodinger equations [7-14].

The concept of fuzzy fractional derivative was introduced by [15] and developed by [16-19], but these researchers tried to put a definition of a fuzzy fractional derivative. Most of them used an integral from the fuzzy fractional derivative. Two of which are the most popular ones, Riemann-Liouville definition and Caputo definition. All definitions mentioned above satisfy the property that the fuzzy fractional derivative is linear. This is the only property inherited from the first fuzzy derivative by all of the definitions. The obtained fractional derivatives in this calculus seemed complicated and lost some of the basic properties that usual derivatives have such as the product rule and the chain rule. However, the semigroup properties of these fractional operators behave well in some cases. Recently, Harir et al. [20] defined a new well-behaved simple fractional derivative called "the fuzzy conformable fractional derivative" depending just on the basic limit definition of the derivative. They proved the product rule and the fractional mean value theorem and solved some (conformable) fractional differential equations [18].

Here, we introduce the fuzzy fractional semigroups of operators associated with the fuzzy conformable fractional derivative, for proving to be a very fruitful tool to solve 
differential equations. Then, we show that this semigroup is a solution to the fuzzy fractional Cauchy problem $x^{(q)}(t)=f(x(t)), x(0)=x_{0}$, and $q \in(0,1]$ according to the fuzzy conformable fractional derivative which was introduced in [20].

\section{Preliminaries}

Let us denote by $\mathbb{R}_{\mathscr{F}}=\{u: \mathbb{R} \longrightarrow[0,1]\}$ the class of fuzzy subsets of the real axis satisfying the following properties [21]:

(i) $u$ is normal, i.e., there exists an $x_{0} \in \mathbb{R}$ such that $u\left(x_{0}\right)=1$,

(ii) $u$ is the fuzzy convex, i.e., for $x, y \in \mathbb{R}$ and $0<\lambda \leq 1$,

$$
u(\lambda x+(1-\lambda) y) \geq \min [u(x), u(y)]
$$

(iii) $u$ is upper semicontinuous,

(iv) $[u]^{0}=\operatorname{cl}\{x \in \mathbb{R} \mid u(x)>0\}$ is compact.

Then, $\mathbb{R}_{\mathscr{F}}$ is called the space of fuzzy numbers. Obviously, $\quad \mathbb{R} \subset \mathbb{R}_{\mathscr{F}}$. For $0<\alpha \leq 1$, denote $[u]^{\alpha}=\{x \in \mathbb{R}$ $\mid u(x) \geq \alpha\}$, then from (i) to (iv), it follows that the $\alpha$-level sets $[u]^{\alpha} \in P_{\mathscr{K}}(\mathbb{R})$, for all $0 \leq \alpha \leq 1$, are a closed bounded interval which we denote by $[u]^{\alpha}=\left[u_{1}^{\alpha}, u_{2}^{\alpha}\right]$.

Here, $P_{\mathscr{K}}(\mathbb{R})$ denotes the family of all nonempty compact convex subsets of $\mathbb{R}$ and defines the addition and scalar multiplication in $P_{\mathscr{K}}(\mathbb{R})$ as usual.

Lemma 1 (see [22]). Let $u, v: \mathbb{R}_{\mathscr{F}} \longrightarrow[0,1]$ be the fuzzy sets. Then, $u=v$ if and only if $[u]^{\alpha}=[v]^{\alpha}$, for all $\alpha \in[0,1]$.

The following arithmetic operations on fuzzy numbers are well known and frequently used below. If $u, v \in \mathbb{R}_{\mathscr{F}}$, then

$$
\begin{aligned}
{[u+v]^{\alpha} } & =\left[u_{1}^{\alpha}+v_{1}^{\alpha}, u_{2}^{\alpha}+v_{2}^{\alpha}\right], \\
{[u-v]^{\alpha} } & =\left[u_{1}^{\alpha}-v_{2}^{\alpha}, u_{2}^{\alpha}-v_{1}^{\alpha}\right], \\
{[\lambda u]^{\alpha} } & =\lambda[u]^{\alpha}= \begin{cases}{\left[\lambda u_{1}^{\alpha}, \lambda u_{2}^{\alpha}\right],} & \text { if } \lambda \geq 0, \\
{\left[\lambda u_{2}^{\alpha}, \lambda u_{1}^{\alpha}\right],} & \text { if } \lambda<0 .\end{cases}
\end{aligned}
$$

For $u, v \in \mathbb{R}_{\mathscr{F}}$, if there exists $w \in \mathbb{R}_{\mathscr{F}}$ such that $u=v+w$, then $w$ is the Hukuhara difference of $u$ and $v$ denoted by $u \ominus v$.

Let us define $d: \mathbb{R}_{\mathscr{F}} \times \mathbb{R}_{\mathscr{F}} \longrightarrow \mathbb{R}^{+} \cup\{0\}$ by the equation

$$
d(u, v)=\sup _{\alpha \in[0,1]} d_{H}\left([u]^{\alpha},[v]^{\alpha}\right), \quad \text { for all } u, v \in \mathbb{R}_{\mathscr{F}},
$$

where $d_{H}$ is the Hausdorff metric defined in $P_{\mathscr{K}}(\mathbb{R})$.

$$
d_{H}\left([u]^{\alpha},[v]^{\alpha}\right)=\max \left\{\left|u_{1}^{\alpha}-v_{1}^{\alpha}\right|,\left|u_{2}^{\alpha}-v_{2}^{\alpha}\right|\right\}
$$

Theorem 1 (see [23]). ( $\left.\mathbb{R}_{\mathscr{F}}, d\right)$ is a complete metric space. We list the following properties of $d(u, v)$ :

$$
\begin{aligned}
& d(u+w, v+w)=d(u, v), \\
& d(u, v)=d(v, u), \\
& d(k u, k v)=|k| d(u, v), \\
& d(u, v) \leq d(u, w)+d(w, v),
\end{aligned}
$$

for all $u, v, w \in \mathbb{R}_{\mathscr{F}}$ and $\lambda \in \mathbb{R}$.

Theorem 2 (see [24]). There exists a real Banach space $X$ such that $\mathbb{R}_{\mathscr{F}}$ can be the embedded as a convex cone $C$ with vertex 0 in $X$. Furthermore, the following conditions hold true:

(i) The embedding $j$ is isometric,

(ii) The addition in $X$ induces the addition in $\mathbb{R}_{\mathscr{F}}$,

(iii) The multiplication by a nonnegative real number in $X$ induces the corresponding operation in $\mathbb{R}_{\mathscr{F}}$,

(iv) $C-C=\left\{a-b / a, b \in \mathbb{R}_{\mathscr{F}}\right\}$ is dense in $X$,

(v) $C$ is closed.

Remark 1. Let $\tilde{j}: \mathbb{R}_{\mathscr{F}} \longrightarrow X$ as $\tilde{j}(u)=j((-1) u), u \in \mathbb{R}_{\mathscr{F}}$. It verifies the following properties: $\|\widetilde{j}(u)-\widetilde{j}(v)\|=d(u, v)$, $\widetilde{j}(s u+t v)=\widetilde{j}(u)+\tau \tilde{j}(v)$, for all $u, v \in \mathbb{R}_{\mathscr{F}}, t, s \geq 0 \widetilde{j}\left(\mathbb{R}_{\mathscr{F}}\right)$ $-j\left(\mathbb{R}_{\mathscr{F}}\right)=C$, since $(-1) \mathbb{R}_{\mathscr{F}}=\mathbb{R}_{\mathscr{F}}$.

\section{Fuzzy $q$-Semigroup of Operators}

Definition 1 (see [20]). Let $F:(0, a) \longrightarrow \mathbb{R}_{\mathscr{F}}$ be a fuzzy function. $q^{\text {th }}$ order "fuzzy conformable fractional derivative" of $F$ is defined by (where the limit is taken in the metric space $\left.\left(\mathbb{R}_{\mathscr{F}}, d\right)\right)$.

$$
\begin{aligned}
T_{q}(F)(t) & =\lim _{\varepsilon \longrightarrow 0^{+}} \frac{F\left(t+\varepsilon t^{1-q}\right) \ominus F(t)}{\epsilon}, \\
& =\lim _{\varepsilon \longrightarrow 0^{+}} \frac{F(t) \ominus F\left(t-\varepsilon t^{1-q}\right)}{\varepsilon},
\end{aligned}
$$

for all $t>0, q \in(0,1)$. Let $F^{(q)}(t)$ stand for $T_{q}(F)(t)$. Hence,

$$
F^{(q)}(t)=\lim _{\varepsilon \longrightarrow 0^{+}} \frac{F\left(t+\varepsilon t^{1-q}\right) \ominus F(t)}{\varepsilon}=\lim _{\varepsilon \longrightarrow 0^{+}} \frac{F(t) \ominus F\left(t-\varepsilon t^{1-q}\right)}{\varepsilon} .
$$

If $F$ is $q$-differentiable in some $(0, a)$ and $\lim _{t \rightarrow 0^{+}} F^{(q)}(t)$ exists, then

$$
F^{(q)}(0)=\lim _{t \longrightarrow 0^{+}} F^{(q)}(t) .
$$


Definition 2. Let $F \in C\left((0, a), \mathbb{R}_{\mathscr{F}}\right) \cap L^{1}\left((0, a), \mathbb{R}_{\mathscr{F}}\right)$. Define the fuzzy fractional integral for $q \in(0,1]$.

$$
I_{q}(F)(t)=I\left(t^{q-1} F\right)(t)=\int_{0}^{t} \frac{F(x)}{x^{1-q}} \mathrm{~d} x
$$

where the integral is the usual Riemann improper integral.

Definition 3. Let $q \in(0, a]$, for any $a>0$. A family $\{T(t)\}_{t \geq 0}$ of operators from $\mathbb{R}_{\mathscr{F}}$ is called a fuzzy fractional $q$-semigroup (or fuzzy $q$-semigroup) of operators if

(i) $T(0)=I$, where $I$ is the identity mapping on $\mathbb{R}_{\mathscr{F}}$,

(ii) $T(s+t)^{(1 / q)}=T\left(s^{(1 / q)}\right) T\left(t^{(1 / q)}\right)$, for all $s, t \geq 0$.

Definition 4. A $q$-semigroup $T(t)$ is called a $c_{0}$-q-semigroup if

(a) The function $g:[0, \infty) \longrightarrow \mathbb{R}_{\mathscr{F}}$, defined by $g(t)=T(t)(x)$, is continuous at $t=0$, for all $x \in \mathbb{R}_{\mathscr{F}}$, i.e.,

$$
\lim _{t \rightarrow 0^{+}} T(t)(x)=x \text {. }
$$

(b) There exist constants $w \geq 0$ and $M \geq 1$ such that $d(T(t) x, T(t) y) \leq M e^{w\left(t^{q} / q\right)} d(x, y)$, for all $t \geq 0, x$, $y \in \mathbb{R}_{\mathscr{F}}$.

Example 1. Define on $\mathbb{R}_{\mathscr{F}}$ the linear operator $T(t) x=e^{2 \sqrt{t}} x$. Then, $\{T(t)\}_{t \geq 0}$ is a fuzzy (1/2)-semigroup. Indeed

(i) $T(0)=I, T(0) x=x$, for all $x \in \mathbb{R}_{\mathscr{F}}$,

(ii) For $t, s \geq 0, x \in \mathbb{R}_{\mathscr{F}}$,

$$
\begin{array}{r}
T(s+t)^{2} x=e^{2 \sqrt{(s+t)^{2}}} x=e^{2(s+t)} x=e^{2 s}\left(e^{2 t} x\right), \\
=T\left(s^{2}\right)\left(e^{2 t} x\right)=T\left(s^{2}\right) T\left(t^{2}\right) x .
\end{array}
$$

(a) For $\quad t, s \geq 0, x \in \mathbb{R}_{\mathscr{F}}, d(T(t) x, x)=d\left(e^{2 \sqrt{t}} x, x\right)$, then $\left(e^{2 \sqrt{t}}-1\right) \geq 0$, and then using Remark 1, we deduce that $\left(e^{2 \sqrt{t}}-1\right) x+x=e^{2 \sqrt{t}} x$. Therefore, the Hukuhara difference $e^{2 \sqrt{t}} x \ominus x(T(t) x \ominus x)$ exists and we have

$$
T(t) x \ominus x=e^{2 \sqrt{t}} x \ominus x=\left(e^{2 \sqrt{t}}-1\right) x .
$$

Then,

$$
\begin{aligned}
d(T(t) x, x) & =d\left(e^{2 \sqrt{t}} x \ominus x, \tilde{0}\right)=d\left(e^{2 \sqrt{t}} x \ominus x, \widetilde{0}\right), \\
& =d\left(\left(e^{2 \sqrt{t}}-1\right) x, \widetilde{0}\right)=\left(e^{2 \sqrt{t}}-1\right) d(x, \widetilde{0}) .
\end{aligned}
$$

Since $\lim _{t \longrightarrow 0^{+}} e^{2 \sqrt{t}}-1=0$, then $\lim _{t \longrightarrow 0^{+}} T(t) x=x$.

(b) For $t \geq 0, x, y \quad \in \mathbb{R} \quad \mathscr{F}, d \quad(T(t) x, T(t) y)=$ $d\left(e^{2 \sqrt{t}} x, e^{2 \sqrt{t}} y\right)=e^{2 \sqrt{t}} d(x, y)$. Consequently, $\{T(t)\}_{t \geq 0}$ is a fuzzy $c_{0}-q$-semigroup on $\mathbb{R}_{\mathscr{F}}$.

Definition 5. The conformable $q$-derivative of $T(t)$ at $t=0$ is called the $q$-infinitesimal generator of the fuzzy $q$-semigroup $\{T(t)\}_{t \geq 0}$, with domain equals

$$
D(A)=\left\{x \in \mathbb{R}_{\mathscr{F}}: \lim _{t \longrightarrow 0^{+}} T^{(q)}(t) x \text { exists }\right\} .
$$

We will write $A$ for such generator.

Lemma 2. Let $A: \mathbb{R}_{\mathscr{F}} \longrightarrow \mathbb{R}_{\mathscr{F}}$ and $A_{1}=j A j^{-1}: C \longrightarrow C$ tow the operator.

$A$ is the operator of the fuzzy q-semigroup $\{T(t)\}_{t \geq 0}$ on $\mathbb{R}_{\mathscr{F}}$ if and only if $A_{1}$ is the operator of the q-semigroup $\left\{T_{1}(t)\right\}_{t \geq 0}$ defining on the convex closed set $C$ and $T_{1}=j T(t) j^{-1}$.

By using Definition 5, the proof is similar to the proof of Lemma 5 in [18] and is omitted.

Theorem 3. Let $\{T(t)\}_{t \geq 0}$ be a $c_{0}$-q-semigroup with infinitesimal generator $A, 0<q \leq 1$. Then, for all $x \in \mathbb{R}_{\mathscr{F}}$ such that $T(t) x \in D(A)$, for all $t \geq 0$; the mapping $t \longrightarrow T(t) x$ is q-differentiable and

$$
T^{(q)}(t) x=\operatorname{AT}(t) x, \quad \forall t \geq 0 .
$$

Proof. Let $q \in(0,1]$ and $x \in \mathbb{R}_{\mathscr{F}}$, for $t \geq 0$, and we have

$$
T(t+s)^{(1 / q)} x=T(t)^{(1 / q)} T(s)^{(1 / q)} x .
$$

Since $T(t) x \in D(A)$, then 


$$
\begin{aligned}
T^{(q)}(t)(x) & =\lim _{\varepsilon \longrightarrow 0} \frac{T\left(t+\varepsilon t^{1-q}\right) x \ominus T(t) x}{\varepsilon}, \\
& =\lim _{\varepsilon \longrightarrow 0} \frac{j^{-1} T_{1}\left(t+\varepsilon t^{1-q}\right) j x-j^{-1} T_{1}(t) j x}{\varepsilon}, \\
& =j^{-1}\left(\lim _{\varepsilon \longrightarrow 0} \frac{T_{1}\left(t+\varepsilon t^{1-q}\right) j x-T_{1}(t) j x}{\varepsilon}\right), \\
& =j^{-1}\left(\lim _{\varepsilon \longrightarrow 0} \frac{T_{1}\left(t^{q}+\left(t+\varepsilon t^{1-q}\right)^{q}-t^{q}\right)^{(1 / q)} j x-T_{1}(t) j x}{\varepsilon}\right), \\
& =j^{-1}\left(\lim _{\varepsilon \longrightarrow 0} \frac{T_{1}(t) T_{1}\left(\left(t+\varepsilon t^{1-q}\right)^{q}-t^{q}\right)^{(1 / q)} j x-T_{1}(t) j x}{\varepsilon}\right), \\
& =j^{-1}\left(\lim _{\varepsilon \longrightarrow 0} \frac{T_{1}(t)\left[T_{1}\left(\left(t+\varepsilon t^{1-q}\right)^{q}-t^{q}\right)^{(1 / q)} j x-T_{1}(0) j x\right]}{\varepsilon}\right) .
\end{aligned}
$$

Now, using Theorem 2.4 in [20], we get

$$
\begin{aligned}
& \frac{\left[T_{1}\left(\left(t+\varepsilon t^{1-q}\right)^{q}-t^{q}\right)^{(1 / q)} j x-T_{1}(0) j x\right]}{\varepsilon} \\
& =T_{1}(t) T_{1}^{(q)}(c) \frac{\left[\left(t+\varepsilon t^{1-q}\right)^{q}-t^{q}\right]}{q \varepsilon} j x,
\end{aligned}
$$

for some $0<c<\left(t+\varepsilon t^{1-q}\right)^{q}-t^{q}$. If $\varepsilon \longrightarrow 0$, then $c \longrightarrow 0$ and $\lim _{\varepsilon \longrightarrow 0} T_{1}^{(q)}(c)=T_{1}^{(q)}(0)=A_{1}$.

Consequently,

$$
T_{1}^{q}(t) j x=T_{1}(t) A_{1} j x \lim _{\varepsilon \rightarrow 0} \frac{\left[\left(t+\varepsilon t^{1-q}\right)^{q}-t^{q}\right]}{q \varepsilon} .
$$

By using L'Hopital's Rule, we get $\lim _{\varepsilon \longrightarrow 0}\left(\left[\left(\left(t+\varepsilon t^{1-q}\right)^{q}-t^{q}\right)\right] / q \varepsilon\right)=1$.

$$
\begin{aligned}
T^{(q)}(t)(x) & =j^{-1}\left(T_{1}(t) A_{1} j x\right), \\
& =j^{-1}\left(A_{1} T_{1}(t) j x\right), \\
& =j^{-1} A_{1} j j^{-1} T_{1}(t) j x, \\
& =\operatorname{AT}(t)(x) .
\end{aligned}
$$

Example 2. Let $f: \mathbb{R}_{\mathscr{F}} \longrightarrow \mathbb{R}_{\mathscr{F}}$ be continuous on $[0,1]$. Define

$$
(T(t) f)(x)=f\left(x+\frac{1}{q} t^{q}\right), \quad q \in(0,1] .
$$

Then, $T(t)$ is obviously a $c_{0}$-q-semigroup of contraction on $\mathbb{R}_{\mathscr{F}}$.

Remark 2. If $M=1$ and $w=0$ in Definition 4 , we say that $\{T(t)\}_{t \geq 0}$ is a contraction fuzzy semigroup.
For $q \in(0,1]$,

$$
\begin{aligned}
\left(T(t+s)^{(1 / q)} f\right)(x) & =f\left(x+\frac{1}{q}\left[(t+s)^{(1 / q)}\right]^{q}\right), \\
& =f\left(x+\frac{1}{q} t+\frac{1}{q} s\right), \\
& =\left(T\left(t^{(1 / q)}\right) T\left(s^{(1 / q)}\right) f\right)(x) .
\end{aligned}
$$

$T(0)=I$ and $T(t) f \in \mathbb{R}_{\mathscr{F}}$ whenever $f \in \mathbb{R}_{\mathscr{F}}$ and that

$$
d(T(t) f, \widetilde{0}) \leq d(f, \widetilde{0}), \quad t \geq 0 .
$$

\section{Fuzzy Fractional Cauchy Problems}

Let $F: \mathbb{R}_{\mathscr{F}} \longrightarrow \mathbb{R}_{\mathscr{F}}$ be continuous and consider the fractional initial value problem

$$
x^{(q)}(t)=F(x(t)), \quad x\left(t_{0}\right)=x_{0},
$$

where $q \in(0,1)$.

It is well known that instead of the differential equation (24), it is possible to study an equivalent fractional integral equation.

$$
x(t)=x_{0}+I_{q} F(x(t)),
$$

for all $t \geq 0$ and $q \in(0,1)$.

A solution $x(t)$ of equation (24) is independent of the initial time $t_{0}$. In fact, let $k_{0}<a$ and denote $y(t)=x\left(k_{0}+(1 / q) t^{q}\right)$. Then, 


$$
y^{(q)}(t)=x^{(q)}\left(k_{0}+\frac{1}{q} t^{q}\right)=F\left(x\left(k_{0}+\frac{1}{q} t^{q}\right)\right)=F(y(t))
$$

and $y\left(t_{0}\right)=x\left(k_{0}+(1 / q) t_{0}^{q}\right)=y_{0}$. Hence, $y(t)$ and $x(t)$ are solutions of the same fractional differential equation with a different initial value.

Theorem 4. Let $q \in(0,1)$.

If $x(t)$ is a solution to the fuzzy fractional initial value problem,

$$
x^{(q)}(t)=F(x(t)), \quad x\left(t_{0}\right)=x_{0} .
$$

Then, $T(t)\left(x_{0}\right)=x(t)$ is a fuzzy semigroup. Furthermore, $T(t)\left(x_{0}\right)$ is q-differentiable w.r.t $t$ and $T^{(q)}(t)\left(x_{0}\right)=F(x(t))$ $=F\left(T(t)\left(x_{0}\right)\right)$.

Proof. Let $q \in(0,1)$ and $k>0$. As obtained above, $y(t)=$ $x\left(k+(1 / q) t^{q}\right)$ is a solution of the fractional initial value problem $y^{(q)}(t)=F(y(t)), y(0)=x(k)$. Hence,

$$
T(s+t)^{(1 / q)}\left(x_{0}\right)=x\left(0+\frac{1}{q}\left((s+t)^{(1 / q)}\right)^{q}\right)=x\left(\frac{1}{q} s+\frac{1}{q} t\right) .
$$

We set $k=(1 / q) s$, then

$$
\begin{aligned}
T(s+t)^{(1 / q)}\left(x_{0}\right) & =x\left(k+\frac{1}{q} t\right)=y\left(t^{(1 / q)}\right)=T\left(t^{(1 / q)}\right) x(k), \\
& =T\left(t^{(1 / q)}\right) x\left(\frac{1}{q} s\right)=T\left(t^{(1 / q)}\right) T\left(s^{(1 / q)}\right)\left(x_{0}\right),
\end{aligned}
$$

and $T(0)\left(x_{0}\right)=x(0)=x_{0}$. Being a solution to a differential equation, $T(t)\left(x_{0}\right)$ is $q$-differentiable with respect to $t$ and $T^{(q)}(t)\left(x_{0}\right)=x^{(q)}(t)=F(x(t))$.

Theorem 5. Let $q \in(0,1]$. Suppose that a fuzzy semigroup $T(t)(x)$ is q-differentiable w.r.t $t$, for all $x \in \mathbb{R}_{\mathscr{F}}$. Then, $T(t)\left(x_{0}\right)$ is a solution to the fractional initial value problem

$$
x^{(q)}(t)=F(x(t)), \quad x\left(t_{0}\right)=x_{0},
$$

where $F(x(t))=T^{(q)}(0)\left(x_{0}\right)$.

Proof. By the $q$-semigroup property and using proof of Theorem 3, we obtain

$$
\begin{aligned}
T^{(q)}(t)\left(x_{0}\right) & =\lim _{\varepsilon \longrightarrow 0} \frac{T\left(t+\varepsilon t^{1-q}\right)\left(x_{0}\right) \ominus T(t)\left(x_{0}\right)}{\varepsilon}, \\
& =\lim _{\varepsilon \longrightarrow 0} \frac{T\left(t^{q}+\left(t+\varepsilon t^{1-q}\right)^{q}-t^{q}\right)^{(1 / q)}\left(x_{0}\right) \ominus T(t)\left(x_{0}\right)}{\varepsilon}, \\
& =\lim _{\varepsilon \longrightarrow 0} \frac{T\left(\left(t+\varepsilon t^{1-q}\right)^{q}-t^{q}\right)^{(1 / q)} T(t)\left(x_{0}\right) \ominus T(t)\left(x_{0}\right)}{\varepsilon}, \\
& =\lim _{\varepsilon \longrightarrow 0} \frac{T\left(\left(t+\varepsilon t^{1-q}\right)^{q}-t^{q}\right)^{(1 / q)} T(t)\left(x_{0}\right) \ominus T(0) T(t)\left(x_{0}\right)}{\varepsilon}, \\
& =T^{(q)}(0) T(t)\left(x_{0}\right),
\end{aligned}
$$

and $T(0)\left(x_{0}\right)=x_{0}$.

Finally, we show that the fuzzy exponential function is a generalization of the fuzzy semigroup introduced in [5].

Theorem 6. If $A: \mathbb{R}_{\mathscr{F}} \longrightarrow \mathbb{R}_{\mathscr{F}}$ is a bounded linear operator, then the fuzzy exponential function has a power series representation

$$
e^{\left(t^{q} / q\right) A}(x)=\sum_{k=0}^{\infty} \frac{t^{k q}}{q^{k} k !} A^{k} x, \quad t \geq 0 .
$$

Proof. Let $A: \mathbb{R}_{\mathscr{F}} \longrightarrow \mathbb{R}_{\mathscr{F}}$ be a bounded linear operator as defined by Gal and Gal in [5]. Then,

$$
\phi(r)=\sup _{d(x, y)<r} d(A x, A y)=r\|A\|
$$

and hence by [6] satisfies the condition. Consequently,

$$
e^{\left(t^{q} / q\right) A}\left(x_{0}\right)=\lim _{n \longrightarrow \infty}\left(I+\frac{t^{q} A}{q n}\right)^{n}\left(x_{0}\right)
$$

is a solution to the Cauchy problem $x^{(q)}(t)=A x(t)$, $x(0)=x_{0}$. Define $S(t)$ by a power series as

$$
S(t)=\sum_{k=0}^{\infty} \frac{t^{k q}}{q^{k} k !} A^{k}
$$

Now, by Theorem 3.9 in [5], $\left(\right.$ pose $\left(s^{q} / q\right)=t$ with $e^{\left(s^{q} / q\right) A}$ and $\left.S(s)\right)$ in [5], so $S(t)$ is a 
fuzzy semigroup, and hence by Theorem $5, S(t)\left(x_{0}\right)$ is a solution to the problem

$$
x^{(q)}(t)=A x(t), \quad x(0)=x_{0} .
$$

Since a bounded linear operator is Lipschitzian, it follows by Theorem 6.1 in [25] that the problem $x^{(q)}(t)=A x(t), x(0)=x_{0}$, has a unique solution. Hence, $e^{\left(t^{q} / q\right) A}\left(x_{0}\right)=S(t)\left(x_{0}\right)$, for all $x_{0} \in \mathbb{R}_{\mathscr{F}}$.

\section{Data Availability}

No data were used to support this study.

\section{Conflicts of Interest}

The authors declare that they have no conflicts of interest.

\section{References}

[1] S. Bochner, "Diffusion equation and stochastic processes," Proceedings of the National Academy of Sciences, vol. 35, no. 7, pp. 368-370, 1949.

[2] A. Balakrishnan, "Fractional powers of closed operators and the semigroups generated by them," Pacific Journal of Mathematics, vol. 10, no. 2, pp. 419-437, 1960.

[3] E. Popescu, "On the fractional cauchy problem associated with a feller semi-group, Romanian academy," Mathematical Reports, vol. 12, no. 62, pp. 181-188, 2010.

[4] T. Abdeljawad, M. Al Horani, and R. Khalil, "Fractional semigroups of operators," Journal of Semigroup Theory and Applications, vol. 2015, p. 7, 2015.

[5] C. S. Gal and S. G. Gal, "Semigroups of operators os spaces of fuzzy-number-valued functions with applications to fuzzy differential equations," Journal of Fuzzy Mathematics, vol. 13, no. 3, pp. 647-682, 2005.

[6] O. Kaleva, "Nonlinear iteration semigroups of fuzzy cauchy problems," Fuzzy Sets and Systems, vol. 209, pp. 104-110, 2012.

[7] F. Anceschi, C. S. Goodrich, and A. Scapellato, "Operators with Gaussian kernel bounds on mixed Morrey spaces," Filomat, vol. 33, no. 16, pp. 5219-5230, 2019.

[8] M. Caputo, "Linear models of dissipation whose $Q$ is almost frequency independent--II," Geophysical Journal International, vol. 13, no. 5, pp. 529-539, 1967.

[9] V. Daftardar-Gejji, Fractional Calculus Theory and Applications, Narosa Publishing House, New Delhi, India, 2013.

[10] K. S. Miller and B. Ross, An Introduction to the Fractional Calculus and Fractional Differential Equations, Wiley and Sons, New York, NY, USA, 1993.

[11] N. Papageorgiou and A. Scapellato, "Nonlinear Robin problems with general potential and crossing reaction," Rendiconti Lincei-Matematica e Applicazioni, vol. 30, no. 1, pp. 1-29, 2019.

[12] N. S Papageorgiou and A. Scapellato, "Constant sign and nodal solutions for parametric $(p, 2)$-equations," Advances in Nonlinear Analysis, vol. 9, no. 1, pp. 449-478, 2020.

[13] M. Ruggieri and M. P. Speciale, "Approximate analysis of a nonlinear dissipative model," Acta Applicandae Mathematicae, vol. 132, no. 1, pp. 549-559, 2014.

[14] Y. Benia, M. Ruggieri, and A. Scapellato, "Exact solutions for a modified Schrödinger equation," Mathematics, vol. 7, no. 10, p. 908, 2019.
[15] R. P. Agarwal, V. Lakshmikantham, and J. J. Nieto, "On the concept of solution for fractional differential equations with uncertainty," Nonlinear Analysis: Theory, Methods \& Applications, vol. 72, no. 6, pp. 2859-2862, 2010.

[16] S. Arshad and V. Lupulescu, "On the fractional differential equations with uncertainty," Nonlinear Analysis: Theory, Methods \& Applications, vol. 74, no. 11, pp. 3685-3693, 2011.

[17] A. Harir, S. Melliani, L. S. Chadli, and E. Minchev, "Solutions of fuzzy fractional heat-like and wave-like equations by variational iteration method," International Journal of Contemporary Mathematical Sciences, vol. 15, no. 1, p. 1135, 2020.

[18] A. Harir, S. Melliani, and L. S. Chadli, "Fuzzy fractional evolution equations and fuzzy solution operators," Advances in Fuzzy Systems, vol. 2019, Article ID 5734190, 10 pages, 2019.

[19] Z. Shuqin, "Monotone iterative method for initial value problem involving Reimann Liouville fractional derivatives," Nonlinear Analysis, vol. 71, no. 5-6, pp. 2087-2093, 2009.

[20] A. Harir, S. Melliani, and L. S. Chadli, "Fuzzy generalized conformable fractional derivative," Advances in Fuzzy Systems, Advances in Fuzzy Systems, vol. 2020, Article ID 1954975, 7 pages, 2019.

[21] P. Diamond and P. E. Kloeden, Metric Spaces of Fuzzy Sets: Theory and Applications, World Scienific, Singapore, Asia, 1994.

[22] H. Y. Goo and J. S. Park, "On the continuity of the Zadeh extensions," Journal of Chungcheong Mathematical Society, vol. 20, no. 4, pp. 525-533, 2007.

[23] M. L. Puri and D. A. Ralescu, "Differentials of fuzzy functions," Journal of Mathematical Analysis and Applications, vol. 91, no. 2, pp. 552-558, 1983.

[24] O. Kaleva, "The cauchy problem for fuzzy differentiel equations," Fuzzy Sets and Systems, vol. 35, pp. 366-389, 1990.

[25] O. Kaleva, "Fuzzy differential equations," Fuzzy Sets and Systems, vol. 24, no. 3, pp. 301-317, 1987. 